\title{
Realidad Virtual como medio de representación de la experiencia especial: Su uso en el diseño participativo.
}

\author{
Constanza Montiel \\ Universidad de Chile, Chile \\ constanza.montiel@gmail.com \\ Mauricio Loyola \\ Universidad de Chile, Chile \\ mloyola@uchile.cl
}

\begin{abstract}
En este estudio comparamos experimentalmente el nivel de comprensión y percepción espacial de un proyecto de arquitectura visualizado usando medios tradicionales versus realidad virtual en un contexto de diseño participativo. Los resultados sugieren que existen diferencias sustantivas entre el tipo de experiencia espacial que se obtiene según el medio de visualización. La realidad virtual ofrece una posición de participante que parece acentuar los aspectos perceptuales de los espacios, detonando respuestas más sensoriales y menos cognitivas sobre el diseño que las otorgadas por los medios tradicionales.
\end{abstract}

Keywords: Realidad virtual; Oculus Rift; Experiencia especial; Diseño participativo.

\section{Introducción}

Numerosos estudios sostienen que las tecnologías de realidad virtual (RV) ofrecen ventajas incomparables para visualizar proyectos de arquitectura pues permiten reproducir las condiciones naturales de percepción del espacio (Angulo 2015, Barria et al 1999, Henry et al 1993, Kalisperis 2006, Westerdahl et al 2006, Witmer y Singer 1998, Daiber 2013). La hipótesis subyacente es que la sensación de presencia inmersiva producida por la RV genera una percepción espacial similar a la experiencia cotidiana en el espacio físico, detonando respuestas perceptuales que serían difíciles de obtener con otros medios de representación arquitectónica tradicionales. Esta diferencia sería especialmente significativa en aquellos usuarios no expertos a quienes los lenguajes de representación arquitectónica les resultan poco familiares.

Sin embargo, no existe suficiente investigación que sustente esta hipótesis. A pesar del explosivo auge que los dispositivos RV de tipo HMD (Head-Mounted Displays) han tenido en el último tiempo, sus aplicaciones en arquitectura son todavía escasas.

Este estudio contribuye a resolver este vacío. Presentamos un experimento que compara la percepción espacial con medios tradicionales versus medios de realidad virtual. El contexto de estudio es un proceso de diseño participativo, un caso paradigmático donde personas no expertas deben visualizar un proyecto de arquitectura y luego hacer comentarios y sugerencias críticas para mejorar el diseño (Schuler y Namioka 1993, Davies 2004, Enet 2007). En el diseño participativo, los medios de representación juegan un papel central, pues influyen directamente en el nivel de comprensión del proyecto que alcancen los participantes, el cual es decisivo en la profundidad de sus comentarios y, por lo tanto, en el éxito de la metodología.

\section{Métodos}

El objetivo específico de este estudio es comparar experimentalmente el nivel de compresión y percepción espacial de un proyecto de arquitectura visualizado usando medios tradicionales (planimetrías y vistas 3D) versus realidad virtual (HMD), en un grupo de usuarios no expertos.

Dos grupos de personas fueron expuestos a la presentación de un proyecto de remodelación urbana y luego se les solicitó opiniones y sugerencias sobre el diseño, replicando un proceso tradicional de diseño participativo. El primer grupo, de control, visualizó el proyecto usando medios tradicionales; el segundo grupo, de estudio, visualizó el proyecto usando equipos de RV. Las respuestas de los participantes fueron capturadas con instrumentos cualitativos y luego analizadas comparativamente.

\section{Caso de estudio}

Como proyecto arquitectónico de estudio se utilizó el proyecto de remodelación urbana "Pasarelas Verdes", ubicado en el conjunto habitacional San Borja en Santiago de Chile, desarrollado por arquitectos y estudiantes de la Universidad de Chile (Universidad de Chile, 2014).

El proyecto propone la rehabilitación y expansión de una serie de pasarelas que conectan las distintas torres del conjunto residencial, las que actualmente se encuentran en abandono y desuso (Figura 1). La propuesta genera un circuito urbanocultural que conecta distintos puntos significativos del sector: el parque San Borja, el barrio Lastarria, el Centro Cultural Gabriela Mistral y el futuro Museo Humano. Además, incorpora la instalación de mobiliario urbano, iluminación, abundante vegetación y programa complementario (café literario, restaurante y huerto comunitario). 


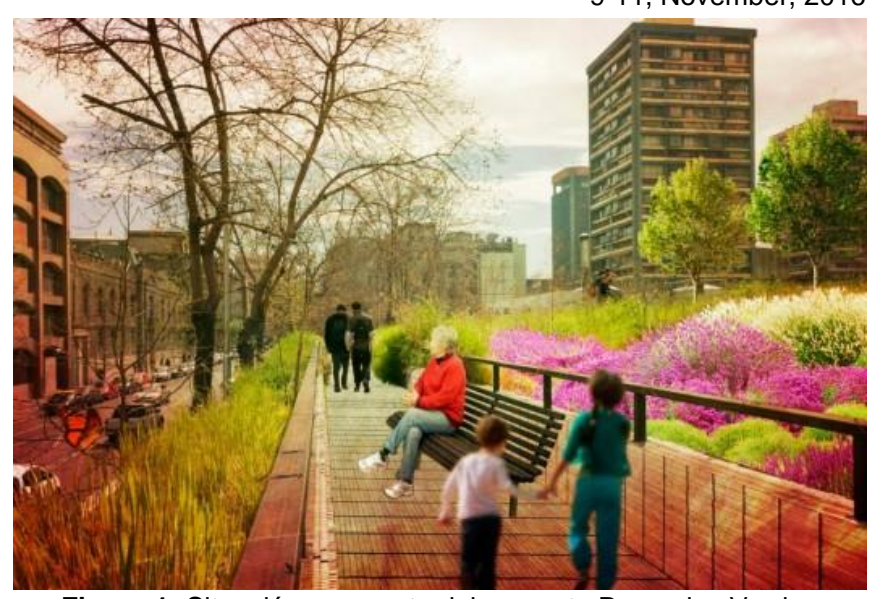

Figura 1: Situación propuesta del proyecto Pasarelas Verdes (Fuente: Equipo Pasarelas Verdes FAU U.Chile)

\section{Muestra}

La muestra fue constituida de manera no probabilística, por conveniencia, utilizando un llamado abierto a los habitantes del conjunto habitacional San Borja. Los participantes seleccionados debían tener capacidades fisiológicas necesarias para utilizar los equipos involucrados (visión normal o corregida a 20/20, sin antecedentes de mareos) y no tener experiencia previa con RV ni conocimiento del proyecto arquitectónico. Se seleccionaron 18 personas para la experiencia, entre 20 y 67 años, de ambos sexos, los que fueron aleatoriamente distribuidos en 2 grupos (control y estudio).

\section{Procedimiento}

El experimento tuvo lugar el día de 17 de agosto entre las 18.00 y 20.30 horas en el Hall Central de la Facultad de Arquitectura y Urbanismo de la Universidad de Chile. La sala se dividió con paneles opacos en 2 zonas independientes, sin conexión visual entre ellos (Figura 2).

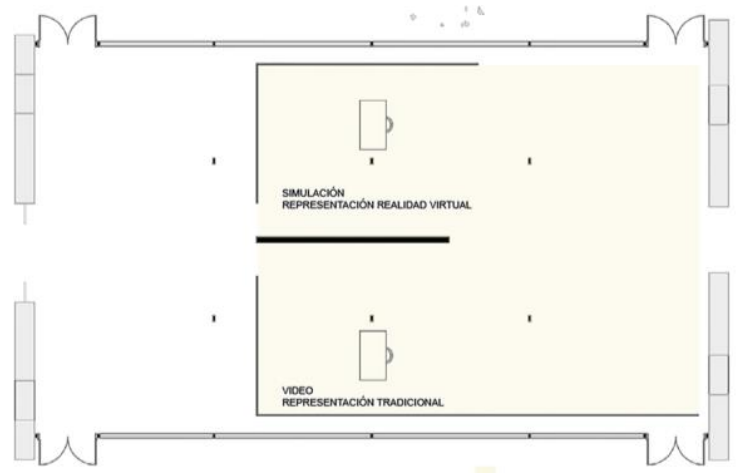

Figura 2: Organización de zonas durante el experimento.

En la zona A se ubicó el grupo de control. Los participantes debieron observar un video de presentación del proyecto de 3 minutos de duración, realizado por el equipo de arquitectos de Pasarelas Verdes. El video, sin audio, mostraba planimetrías y un recorrido de un modelo $3 \mathrm{D}$ del proyecto desarrollado en SketchUp.
En la zona B se ubicó el grupo de estudio. Como instrumento de VR se utilizó un equipo Oculus Rift DK2 y un joystick PlayStation 2 para navegar en el espacio virtual. Se construyó un modelo de RV del proyecto usando como base el mismo modelo 3D desarrollado en SketchUp, pero post-procesado con el motor de juegos Unreal Engine para habilitar la navegación en primera persona. En otras palabras, el modelo de RV contenía exactamente la misma información visual que el modelo 3D usando en el grupo de control. A los participantes primero se les presentó el equipo y su modo de utilización, y luego se les solicitó que recorrieran virtualmente el proyecto con total libertad durante un periodo de 3 minutos.

Durante ambos procesos de visualización, no se aceptaron preguntas relacionadas con el proyecto ni tampoco comentarios cruzados entre los participantes. La Figura 3 muestra fotografías de ambos escenarios experimentales.
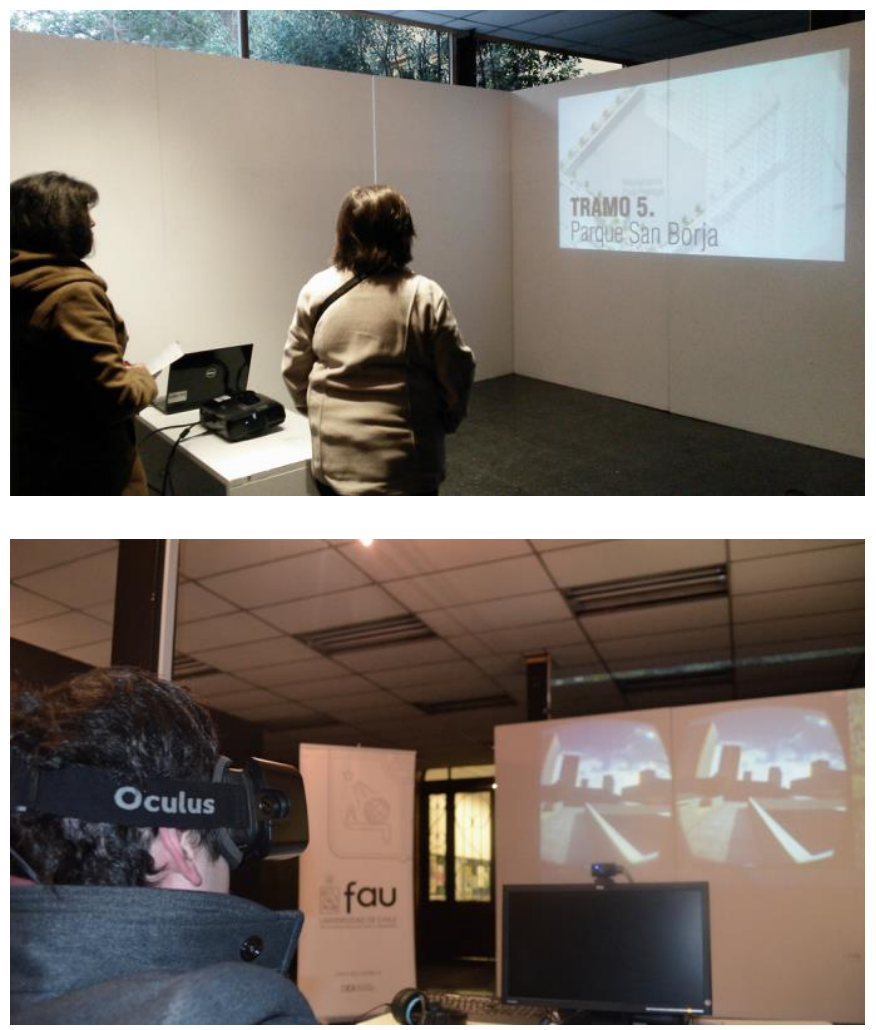

Figura 3, Arriba: Zona A (M. Trad) Abajo: Zona B (RV).

\section{Instrumentos}

Las respuestas de los participantes fueron capturadas con dos instrumentos cualitativos: un cuestionario escrito (dividido en dos partes) y una entrevista estructurada.

La primera parte del cuestionario tenía el propósito de medir la comprensión espacial del proyecto, es decir, la fidelidad y precisión con que el participante comprende la organización, elementos componentes y características espaciales del proyecto. Se incluyeron 4 preguntas cerradas sobre aspectos del proyecto, de carácter factual, cuyas respuestas eran 
absolutas y, por consiguiente, podían ser evaluadas como correctas o incorrectas. La tabla 1 muestra las preguntas del cuestionario y sus respuestas esperadas. La segunda parte del cuestionario estaba destinada a medir la respuesta perceptual y evaluación subjetiva por partes de los participantes. Se incluyeron 5 afirmaciones sobre supuestas características del proyecto y se solicitó a los respondientes manifestar su grado de acuerdo o desacuerdo en una escala de Likert de 9 niveles (Tabla 2). A diferencia del cuestionario anterior, en este instrumento no existen respuestas inherentemente correctas.

Tabla 1: Cuestionario de medición de comprensión espacial

\begin{tabular}{|ll|}
\hline Pregunta & Respuestas esperadas \\
\hline $\begin{array}{l}\text { ¿Cuáles son los nuevos lugares } \\
\text { propuestos en el proyecto? }\end{array}$ & $\begin{array}{l}\text { Huerto comunitario; café } \\
\text { literario; nuevas pasarelas }\end{array}$ \\
\hline ¿De qué manera se conecta el & Por la extensión de las \\
parque San Borja con el & pasarelas del conjunto \\
proyecto? & habitacional \\
\hline ¿Qué actividades de & Paseos; tocatas/conciertos; \\
esparcimiento se pueden realizar & teatro callejero; cultivo de \\
en los nuevos espacios del & vegetales; ferias (y \\
proyecto? & similares). \\
\hline ¿Cuáles son los elementos & Cruce peatonal en Alameda \\
propuestos por el proyecto para & frente al GAM; conexión \\
fomentar un mejor acceso a las & con el Metro; extensión de \\
pasarelas? & pasarelas al parque. \\
\hline
\end{tabular}

Tabla 2: Cuestionario de medición de evaluación subjetiva.

\section{Pregunta}

El proyecto mejora la seguridad del espacio público.

El proyecto invita al descanso y al ocio.

El proyecto es atractivo visualmente.

El proyecto se relaciona adecuadamente con el barrio.

El proyecto mantiene una buena distancia entre actividades.

La entrevista estructurada incluyó 5 preguntas abiertas destinadas a recoger comentarios y sugerencias de diseño, de manera similar a lo que se realiza en un proceso de participación ciudadana (Tabla 3).

Tabla 3: Cuestionario de entrevista estructurada

\section{Pregunta}

¿Qué elementos o espacios del proyecto no le parecen necesarios?

¿Qué elementos o espacios del proyecto le parecen positivos? ¿Qué sugerencias tiene respecto a los siguientes espacios: huerto comunitario, locales comerciales, accesos públicos? ¿Qué opinión tiene respecto a la conexión del proyecto con el parque San Borja? ¿Qué beneficios o inconvenientes le ve? ¿Qué elementos arquitectónicos le agregaría usted al proyecto?

\section{Resultados}

En general, los resultados muestran un nivel de comprensión espacial similar entre ambos grupos, con una posible superioridad de los medios tradicionales. En cambio, los resultados de sugerencias de diseño muestran una evidente y marcada diferencia entre ambos grupos.

En relación a las preguntas de comprensión espacial, en total, el grupo de control (medios tradicionales, MT) obtuvo un $72 \%$ de respuestas correctas versus un $56 \%$ obtenido por el grupo de estudio $(\mathrm{RV})$. El desglose de respuestas correctas por pregunta es el siguiente:

$$
\begin{aligned}
& \mathrm{P} 1(\mathrm{MT}=89 \% \mid \mathrm{RV}=67 \%) ; \\
& \mathrm{P} 2(\mathrm{MT}=67 \% \mid \mathrm{RV}=33 \%) ; \\
& \mathrm{P} 3(\mathrm{MT}=89 \% \mid \mathrm{RV}=67 \%) ; \\
& \mathrm{P} 4(\mathrm{MT}=44 \% \mid \mathrm{RV}=56 \%) .
\end{aligned}
$$

Los MT aparecen superiores para comunicar los aspectos generales del proyecto, sin embargo, estos resultados deben ponderarse con cuidado. Un análisis fino de las repuestas muestra que, en el caso de la RV, algunas de las respuestas "incorrectas" se explican porque algunos usuarios no tenían suficiente información de la totalidad del proyecto ya que no habían visitado todos los espacios en su recorrido virtual libre. Controlando por este factor, los valores entre ambos grupos quedan muy cercanos ( $M T=72 \% \mid R V=69 \%$ ), lo que sugiere que la comprensión espacial es similar en ambos casos. Tampoco existen diferencias apreciables en el lenguaje, intensidad, o tiempo de respuesta entre ambos grupos.

Los resultados de las preguntas de evaluación subjetiva son prácticamente idénticos entre ambos grupos y no permiten detectar ninguna tendencia o diferencia significativa.

Las diferencias más reveladoras entre ambos grupos se encuentran en el análisis de las sugerencias de diseño.

En el grupo de control, las respuestas perceptuales fueron neutras y la evaluación del proyecto tendió hacia la conformidad, sin críticas. Los comentarios y sugerencias fueron sobre aspectos conceptuales, organizacionales 0 administrativos del proyecto, sin mención a características de diseño. En el grupo de estudio, por el contrario, las respuestas perceptuales fueron matizadas y la evaluación del proyecto tuvo mayor crítica. Notablemente, los comentarios y sugerencias se relacionaron mayoritariamente a características del diseño, tales como dimensiones, espacialidad, materiales, condiciones de accesibilidad 0 atractivo visual.

A continuación se muestran extractos de algunas de las respuestas del grupo de control y estudio, en las que se vislumbran las diferencian:

Grupo de estudio:

"[Las terrazas] me parece que son demasiado extensas, es un espacio para nada más que transitar por lo que está ocupando un espacio muy grande".

"En la parte de arriba del supermercado sería bueno agregarle 
una protección al sol porque es una explanada grande donde se pueden hacer hartas actividades".

"Hay una escalera que sube en la parte de las jardineras; entiendo que es para entrar más cómodamente, pero no estoy seguro si ahí debería estar la escalera".

"He visto que hay bancas en líneas que no permiten que un grupo se pueda sentar".

Grupo de control:

"yo creo que [el proyecto] está bien, no se me ocurre otra cosa".

"Creo que [el proyecto] es un beneficio (...) porque el hecho de transitar más gente va a permitir también que el parque sea más accesible (sic)"

"[el problema] de los graffitis que están incorporados en las pasarelas: ¿Cómo se van a mantener?”

"Me gustaría que se pudieran hacer talleres o actividades culturales".

Esta aparente diferencia entre las respuestas fue posteriormente corroborada con un análisis de contenido textual. Las respuestas fueron codificadas y categorizadas de la siguiente forma:

Para las respuestas relativas a evaluación del proyecto:

- Conforme: Respondiente se encuentra de acuerdo o satisfecho con lo expuesto/consultado

- No Conforme: Respondiente se encuentra en desacuerdo o insatisfecho con lo expuesto/consultado

- Interrogante: Respondiente tiene dudas, sin lograr una opinión clara o absoluta sobre lo expuesto/consultado

- Sin Información: Respondiente declara no tener conocimiento sobre lo expuesto/consultado

Para las respuestas relativas a sugerencias de proyecto, denominadas aquí "Requerimientos":

- $\quad$ Req. de Accesibilidad, relacionados con la facilidad de acceso y circulación, especialmente para personas de movilidad reducida.

- Req. de Diseño, relacionados con la forma, tamaños, proporciones o materialidad de los distintos componentes y espacios del proyecto

- Req. de Equipamiento, relacionados con la inclusión de mobiliario y servicios públicos en el proyecto

- $\quad$ Req. de Usos, relacionados con la presencia de espacios específicos para acoger determinados usos o actividades

- Req. de Planificación, relacionados con los procedimientos de planificación del proyecto en general

- Req. de Operación, relacionados con las condiciones de operación del proyecto durante su eventual uso

En el grupo de control (medios tradicionales), las respuestas de evaluación son contundentes: en todas las preguntas realizadas la categoría "Conforme" tuvo resultados mayoritarios (70\%) sin excepción. Respecto a sugerencias de proyecto, la mayor cantidad de respuestas correspondieron a "Requerimientos de usos", los que en su mayoría se referían a la solicitud de espacios para acoger en las pasarelas la realización de actividades socio-culturales que hoy realizan en otros sectores del barrio, lo que supone que el respondiente basó su sugerencia en conocimiento previo del sector y no necesariamente en base a lo visto en la presentación del video. La categoría que le sigue en cantidad de comentarios es "Req. de operación". Comentarios relativos a la materialidad o proporciones de los espacios en el proyecto no son mencionados por ninguno de los usuarios, de manera que la categoría "Requerimientos de diseño" obtiene valor nulo.

En el grupo de estudio (realidad virtual), las respuestas sobre evaluación aparecen como más balanceadas entre "Conforme" y "No conforme" (51\% y $22 \%$ respectivamente), y se aprecia una mayor proporción de "Interrogante" y "Sin Información” (27\%). Respecto a sugerencias de diseño, se observa que no existe grandes brechas entre los valores de las diferentes categorías, con una ligera mayor frecuencia de "Req. de usos" y "Req. de diseño". Llama la atención la variedad de comentarios y requerimientos, con prácticamente ninguna sugerencia repetida, incluyendo, entre otros, inexistencia de espacios de sombra, materiales utilizados, conexiones visuales entre distintos puntos, problemas de evacuación de aguas lluvias, atractivo visual de zonas específicas del proyecto o incluso criterios de mantención del huerto comunitario.

\section{Discusión}

Los resultados de este experimento sugieren que existen diferencias sustantivas entre el tipo de percepción espacial generada entre los grupos de representación de medios tradicionales y realidad virtual.

La posición de observador ofrecida por los medios tradicionales facilita una mejor comprensión de la organización general del proyecto, pero limita la percepción de aspectos de diseño espacial. Esto se evidencia en las respuestas obtenidas de las entrevistas, donde la mayoría corresponden a la categoría de "Conforme" y las sugerencias más frecuentes se refieren a aspectos de uso y operación. Por el contrario, no se realizan sugerencias o comentarios sobre aspectos de diseño.

La posición de participante ofrecida por la realidad virtual acentúa los aspectos perceptuales de los espacios, detonando respuestas más sensoriales y menos cognitivas sobre el diseño. Las evaluaciones del proyecto fueron más matizadas y críticas que en el grupo de control, suponiendo una mayor o más profunda reflexión sobre las características del espacio. Un ejemplo claro de ello fueron las respuestas de Requerimientos de Accesibilidad, obtenidas únicamente en los cuestionarios del grupo de estudio, evidenciando una carencia de rampas para discapacitados, lo que, en contraste, pasó desapercibido en el grupo de control.

Un aspecto que merece mayor análisis es la aparente superioridad de los medios tradicionales para comunicar información completa e integral del proyecto, lo que se manifiesta en los mejores resultados de la medición de compresión espacial y en el menor número de respuestas de 
"Interrogante" y "Sin Información" en la evaluación de proyecto. Una posible explicación es que, en este experimento, los participantes del grupo de realidad virtual recorrieron libremente el espacio, dando cabida a lugares no visitados virtualmente, lo que explicaría la falta de conocimiento total del proyecto. Otra explicación posible de argumentar, es que las vistas generales otorgadas por las planimetrías facilitan la comprensión lógica de la totalidad del proyecto, mientras que, en contraste, la RV ofrece una aproximación experiencial y secuencial de los espacios que dificulta la creación de mapas mentales globales del proyecto.

Este estudio parece indicar que, en el contexto del diseño participativo, las tecnologías de realidad virtual ofrecen ventajas en pertinencia y aplicabilidad de los comentarios recibidos para retroalimentar el diseño. En efecto, las respuestas obtenidas de los cuestionarios del grupo RV dan cuenta que los usuarios generaron nuevos requerimientos e ideas para el proyecto. Esto se presenta como una cualidad sumamente rescatable y esencial de la tecnología para poder ser utilizada en procesos de participación ciudadana, ya que permitiría generar proyectos arquitectónicos y urbanos en base a una participación más activa, participativa y representativa de las necesidades de los usuarios.

\section{Conclusiones}

Este estudio comparó experimentalmente el nivel de compresión y percepción espacial de un proyecto de arquitectura visualizado usando medios tradicionales (planimetrías y vistas 3D) versus realidad virtual (HMD), en un grupo de usuarios no expertos (diseño participativo).

Los resultados parecen indicar que existen diferencias sustantivas entre el tipo de percepción espacial generada según el método de visualización arquitectónica. La realidad virtual ofrece una posición de participante que parece acentuar los aspectos perceptuales de los espacios, detonando respuestas más sensoriales y menos cognitivas sobre el diseño que las otorgadas por los medios tradicionales.

Sin embargo, estos resultados preliminares deben ser validados con un experimento de mayor alcance y profundidad.

En general, este estudio contribuye a la identificación de una fortaleza específica de la realidad virtual en la representación de proyectos, lo que permite orientar la investigación, desarrollo y aplicación práctica de la tecnología.

\section{Agradecimientos}

A la Facultad de Arquitectura y Urbanismo de la Universidad de Chile por acoger y apoyar esta investigación.

A Max Daiber por la modelación y operación del modelo de realidad virtual. A Henry Bauer y al equipo de Pasarelas Verdes por su sustancial colaboración. A Viviana Fernández, Emmanuel Farías, Luna Benavente y Javier Ríos por su orientación sobre los procesos de diseño participativo.

\section{Referencias}

Angulo, A. (2015). Rediscovering Virtual Reality in the Education of Architectural Design: The immersive simulation of spatial experiences.Ambiances. Environnement sensible, architecture et espace urbain.

Barría Chateau, H., García Alvarado, R., Lagos Vergara, R., \& Parra Márquez, J. C. (1999). Evaluación de la percepción espacial en ambientes virtuales. In Libro Ponencias V Congreso Iberoamericano Gráfica Digital, UBio-Bio, Concepción (pp. 145151).

Daiber, M. (2013). Critica a los medios de representación en el proceso de diseño: teoría de la telepresencia como criterio para abordar el proyecto de arquitectura. Seminario de Investigación, Universidad de Chile, Facultad de Arquitectura y Urbanismo, Santiago.

Davies, R. C. (2004). Adapting virtual reality for the participatory design of work environments. Computer Supported Cooperative Work (CSCW), 13(1), 1-33.

Enet, M. (2007). Diseño participativo: Una herramienta de la Producción Social del Hábitat.

Henry, D., \& Furness, T. (1993, September). Spatial perception in virtual environments: Evaluating an architectural application. In Virtual Reality Annual International Symposium, 1993., 1993 IEEE (pp. 33-40). IEEE.

Hernández, L., Taibo, J., Seoane, A., \& Jaspe, A. (2011). Space perception in architectural visualization through immersive virtual reality. Revista de EGA, (18).

Kalisperis, L., Muramoto, K., Balakrishnan, B., Nikolic, D., \& Zikic, N. (2006). Evaluating relative impact of virtual reality system variables on architectural design comprehension and presence. Proceedings of eCAADe2006, Volos, Greece.

Schuler, D., \& Namioka, A. (Eds.). (1993). Participatory design: Principles and practices. CRC Press.

Westerdahl, B., Suneson, K., Wernemyr, C., Roupé, M., Johansson, M., \& Allwood, C. M. (2006). Users' evaluation of a virtual reality architectural model compared with the experience of the completed building. Automation in construction, 15(2), 150-165.

Witmer, B. G., \& Singer, M. J. (1998). Measuring presence in virtual environments: A presence questionnaire. Presence: Teleoperators and virtual environments, 7(3), 225-240. 